\title{
Inverse spectral problem for Jacobi operators and Miura transformation
}

https://doi.org/10.1515/conop-2020-0116

Received March 19, 2021; accepted May 28, 2021

\begin{abstract}
We study a Miura-type transformation between Kac - van Moerbeke (Volterra) and Toda lattices in terms of the inverse spectral problem for Jacobi operators, which appear in the Lax representation for such systems. This inverse problem method, which amounts to reconstruction of the operator from the moments of its Weyl function, can be used in solving initial-boundary value problem for both systems. It is shown that the Miura transformation can be easily described in terms of these moments. Using this description we establish a bijection between the Volterra lattices and the class of Toda lattices which is characterized by positivity of Jacobi operators in their Lax representation. Also, we discuss an implication of the latter result to the spectral theory.
\end{abstract}

Keywords: Jacobi operators, Positive operators, Deficiency indices, Power moment problem, Toda lattices, Volterra lattices, Miura transformations

MSC: 44A60, 47B36, 37K10, 37K15

\section{Introduction}

In 1967, C. Gardner, J. Greene, M. Kruskal and R. Miura discovered a remarkable method for solving the Cauchy problem for the Korteweg-de Vries equation

$$
u_{t}=-u_{x x x}+6 u u_{x}
$$

by using the inverse scattering problem for one-dimensional Schrödinger (or Sturm-Liouville) operator on the line [7]. Since then, the integration of nonlinear equations by using various inverse problems methods is among the main topics in modern mathematical physics. This integration task has inspired the development of various aspects of the theory of differential and difference operators, as well as the areas of mathematics related to inverse problems.

In particular, since the works of Kac, van Moerbeke and Moser [13, 14] the inverse spectral problems for difference operators have found a wide application for integration of nonlinear dynamical systems (also called nonlinear lattices). These inverse problems are connected with the theory of continued fractions, moment problems, Hermite-Pade approximations and orthogonal polynomials, so the development of integration methods for nonlinear lattices had an influence on these areas [3, 6, 17].

Often the operators used for the integration purpose possess some specific properties (e. g. part of their coefficients are zero). The inverse problems for such operators belong to the class of incomplete ones. The characteristic property of this class is that part of the information about the object to reconstruct is known a priori, see [24] and references thereafter. The solution of incomplete inverse problems is generally more complicated than that of their "complete" counterparts, because in this case some additional conditions, providing the existence of a priori known properties of the reconstructed operator, must be established.

${ }^{\star}$ Corresponding Author: Andrey Osipov: Federal State Institution, "Scientific-Research Institute for System Analysis, of the Russian Academy of Sciences", Nakhimovskii pr. 36-1, Moscow, 117218, Russia, E-mail: osipa68@yahoo.com 
Turning back to the nonlinear equations, we note that an important role in their study is played by various Bäcklund-type transformations which relate the equations and their solutions. In particular, the original Miura transformation (which can be regarded as a special case of Bäcklund transformation)

$$
M t: v \rightarrow u \quad u=v_{x}+v^{2},
$$

establishes a relation between the modified Korteweg-de Vries equation

$$
v_{t}=-v_{x x x}+6 v^{2} v_{x}
$$

and the KdV itself. Such transformations can be used for constructing new solutions out of the known ones (for example, using the modified Miura transformations $u=v_{X}+v^{2}+c$, where $c$ is a real constant, one may get a soliton solution of the KdV equation out of the trivial one $v=0$ ). Also, these transformations are useful in the study of the Hamiltonian properties of integrable systems. For instance, one can check the existence of the Poisson bracket of the modified Korteweg-de Vries equation, pushed forward by $M t$ to the Gardner-ZakharovFaddeev bracket [25] of the KdV and having the same form as the latter, see [21] Chapter 3 for details.

Our purpose is to study some links between the inverse spectral problems and the Miura-type transformations. We consider two classical nonlinear lattices: the Kac - van Moerbeke lattice (also known due to its various applications as Volterra lattice, Langmuir lattice or discrete KdV equation [12-14, 16]) and the Toda lattice, and a discrete Miura transformation between them. Both these systems are integrable by the inverse spectral problem method for Jacobi operators which appear in their Lax representations (its brief description for the semi-infinie Kac - van Moerbeke lattice is contained in the original paper [13]; a detailed description of the method and its application to the semi-infinite Toda lattice is contained in the paper of Berezanski [5]). Here we study the inverse spectral problem for positive Jacobi operators. Note that since the positiveness of the operator is a priori known, this inverse problem can be regarded as incomplete. We establish a criterion for its solvability in terms of the inverse spectral data. Also, as noted in [19], the Miura transformation between Volterra and Toda lattice hierarchies (even in case of non-Abelian lattices) can be described as a transformation between the inverse spectral data corresponding to the two systems. Combining these results, we find below that this transformaion is equivalent to the Miura transformation and establishes a one-to-one correspondence between the Volterra lattices and the Toda lattices with positive Jacobi operators.

The paper is organized as follows. In the next section we consider the inverse spectral problem for positive Jacobi operators and obtain a criterion for its solvability. In Section 3, we study the above-mentioned Miura transformation and its description in terms of the inverse spectral data of Jacobi operators. In the final section, we discuss the obtained results and their possible applicability to nonlinear dynamics and spectral theory of Jacobi operators.

\section{Inverse spectral problem for positive Jacobi operators.}

\subsection{Inverse spectral problem}

At the beginning we recall several basic facts on Jacobi operators needed for our further considerations (for more details see e. g. [4] Chapter VII and [1]).

Namely, consider the semi-infinite Jacobi matrix

$$
J=\left(\begin{array}{cccc}
b_{0} & a_{0} & 0 & 0 \\
a_{0} & b_{1} & a_{1} & 0 \\
0 & \ddots & \ddots & \ddots
\end{array}\right)
$$

such that

$$
a_{i}>0, b_{i} \in \mathbb{R}, \quad i \in \mathbb{Z}_{+} .
$$


Denote by $l^{2}=l^{2}[0, \infty)$ the Hilbert space of complex quadratic summable sequences $y=\left(y_{n}\right)_{n=0}^{\infty}$, such that $\sum_{n=0}^{\infty}\left|y_{n}\right|^{2}<\infty$, with the inner product $(y, z)=\sum_{n=0}^{\infty} y_{n} \bar{z}_{n}$, and by $\left(e_{n}\right)_{n=0}^{\infty}$ its standard orthonormal basis. Define $D_{0}^{\prime} \in l^{2}[0, \infty)$ as the dense linear set consisting of finitely nonzero vectors, and $D_{1}$ as the set of vectors $y$ such that $J y \in l^{2}[0, \infty)$. Denote by $J^{\prime}$ the operator defined on $D_{0}^{\prime}$ via matrix multiplication: $J^{\prime} y=J y$. One can check (see e. g. [18], Section 3) that for $y, z \in D_{0}^{\prime}$ )

$$
\left(J^{\prime} y, z\right)-\left(y, J^{\prime} z\right)=0 .
$$

Thus, $J^{\prime}$ is a symmetric operator with a dense domain. Therefore, it admits closure, for which we keep the same notation as for the original matrix $J$. Denote its domain as $D(J)$ (for its description, see [1], [9], [18]). Obviously, in the basis $\left(e_{n}\right)$ this operator has the matrix representation $J$. The deficiency indices of $J$ are either $(0,0)$ or $(1,1)$; it is said that the limit-point or limit-circle case holds for $J$. In the limit-point case $J=J^{\star}$, where the adjoint of $J$ is the operator defined on the set $D_{1}$ in the same manner as $J^{\prime}$. The operators $J$ and $J^{\star}$ are often called, respectively, the minimal (closed symmetric) and the maximal Jacobi operators generated by the matrix $J$. We say that $J$ is a positive operator if

$$
(J y, y) \geq 0, \quad \forall y \in D(J) .
$$

The concept of a spectral function of symmetric operators plays an important role in their study. It appeared as a result of the theory of generalized extensions of symmetric operators developed by Naimark and Akhiezer, (see [15], [2] and references thereafter). In particular, this theory allows one to construct self adjoint extensions of a given symmetric operator with arbitrary (not necessarily equal to each other) deficiency indices, with exit from the Hilbert space where the operator acts, to a larger one. As was rightly noted in [1] "the theory of operators lacks a unified terminology and a unified notation", so we give here the necessary definitions.

A generalized resolution of the identity is defined as a one parameter family of operators $F_{t}, t \in \mathbb{R}$ which satisfy the following conditions:

1. Each difference $F_{t_{2}}-F_{t_{1}}$, where $t_{2}>t_{1}$ is a bounded positive operator,

2. $F_{t-0}=F_{t}$ for all real $t$,

3. $F_{-\infty}=O, F_{\infty}=E$, where $O$ and $E$ are the zero and identity operators respectively.

Unlike in the definition of the ordinary resolution of the identity, it is not assumed here that $F_{t}$ are orthogonal projection operators.

A generalized resolution of the identity $F_{t}$ is called a spectral function for the symmetric operator $A$ acting in a Hilbert space $H$ with a domain of definition $D(A)$ dense in $H$, if for arbitrary elements $f \in D(A)$ and $g \in H$ the following formulas hold:

$$
\begin{aligned}
& (A f, g)=\int_{-\infty}^{\infty} \lambda d\left(F_{\lambda} f, g\right) ; \\
& \|A f\|^{2}=\int_{-\infty}^{\infty} \lambda^{2} d\left(F_{\lambda} f, f\right) .
\end{aligned}
$$

Each symmetric operator has at least one spectral function. If $A$ is self-adjoint, then its spectral function is unique and coincides with its spectral measure. From this definition follows that if $\Delta=\left(\lambda_{1}, \lambda_{2}\right)$ is a finite real interval, and if $g$ is an arbitrary vector in $H$, then for each $h \in H, E(\Delta) g=\left(F_{\lambda_{2}}-F_{\lambda_{1}}\right) g \in D\left(A^{\star}\right)$ and

$$
\left(A^{\star} E(\Delta) g, h\right)=\int_{\Delta} \lambda d\left(F_{\lambda} g, h\right) ;
$$

where $D\left(A^{\star}\right)$ is the domain of the adjoint of $A$ (for the proof see [2], Appendix 1).

We now turn back to the case of Jacobi operators. For an operator $J$ all vectors $J^{k} e_{0}, k \in \mathbb{Z}_{+}$belong to its domain and the linear hull of these vectors is dense in $l^{2}$ since it coincides with the linear hull of the basis 
$\left\{e_{k}\right\}$. Thus, $e_{0}$ is a cyclic vector of the operator $J$. Denote as $\rho(\lambda)$ the function $\left(E_{\lambda} e_{0}, e_{0}\right)$, where $E_{\lambda}$ is a spectral function of $J$. Note that an important role in the inverse spectral theory or differential and difference operators (especially in the non-symmetric case) is played by their Weyl functions, [24]. Here, the Weyl function of $J$ is defined as the Stieltjes transform of $\rho(\lambda)$ :

$$
m(z)=\int_{-\infty}^{\infty} \frac{d \rho(\lambda)}{\lambda-z}, \quad z \in \mathbb{C} .
$$

In terms of the inverse spectral problems, $m(z)$ is the object equivalent to $\rho(\lambda)$. The inverse spectral problem for the operator $J$ can be formulated as follows: given $\rho(\lambda)$ or $m(z)$, find $a_{i}$ and $b_{i}$ (restore the matrix $J$ ). Since $J^{k} e_{0} \in D(J)$ for all $k$, it can be checked, using the definition of the inverse spectal function, (see e. g. [1]) that

$$
s_{k}:=\left(J^{k} e_{0}, e_{0}\right)=\int_{-\infty}^{\infty} \lambda^{k} d \rho(\lambda) .
$$

Note that since $E_{\lambda}$ is not the ordinary resolution of the identity, establishing (5) requires using the properties of spectral functions of symmetric operators, see [1, 2]. It follows from (5) that for an arbitrary polynomial $p(\lambda)$ :

$$
\left(p(J) e_{0}, e_{0}\right)=\int_{-\infty}^{\infty} p(\lambda) d \rho(\lambda) .
$$

Thus, the quantities $s_{k}$ are the moments of $\rho(\lambda)$ which, as follows from (5), can be calculated by the formula

$$
s_{k}=\left(J^{k}\right)_{0,0}, \quad k \in \mathbb{Z}_{+},
$$

where the latter notation is for the matrix $J$ (here we assume using the matrix representation of the identity operator in $l^{2}$ that $\left.1=(E)_{0,0}=s_{0}=\int_{-\infty}^{\infty} d \rho(\lambda)\right)$. Denote as $S=\left\{s_{j}\right\}_{j=0}^{\infty}$ the moment sequence of $\rho(\lambda)$. Due to equivalence between the latter and the Weyl function, and the (formal) asymptotic expansion at infinity

$$
m(z) \sim-\frac{s_{0}}{z}-\frac{s_{1}}{z^{2}}-\ldots,
$$

the sequence $S$ may also be called the moment sequence of $m(z)$. It is important to note that although the operator $J$ may have different spectral functions (and therefore different functions $\rho(\lambda)$ and $m(z)$ ), the sequence $S$ is unique for the matrix (and for the operator) $J$ and is defined by (7). In some sources it is also called the moment sequence associated with $J$.

We see that a solution of the power (Hamburger) moment problem for $S$ can be given by $\rho(\lambda)$. Moreover, if $\sigma(\lambda)$ is another solution for the sequence $S$ given by (7), then it can be shown (see e. g. [1] Theorem 4.1.3) that there exists a spectral function $F_{\lambda}$ of $J$ such that $\sigma(\lambda)=\left(F_{\lambda} e_{0}, e_{0}\right)$. In other words, each solution of the moment problem for $S$ can be described via spectral functions of $J$.

According to the Hamburger's theorem, $S$ is a positive sequence which means that for all $n$

$$
\Delta_{n}(J)>0
$$

where $\Delta_{n}(J)$ are determinants of the Hankel matrices $H_{n}=\left(s_{i+j}\right)_{i, j=0}^{n}$. The positiveness of $S$ implies that a corresponding $\rho(\lambda)$ defined on $\mathbb{R}$ has infinitely many growth points. Also, $\rho(\lambda)$ is a non-decreasing function as follows from its definition.

The following second-order difference equation is assigned to the matrix $J$ :

$$
a_{n-1} y_{n-1}+b_{n} y_{n}+a_{n} y_{n+1}=\lambda y_{n}, \lambda \in \mathbb{C}, \quad n=1,2, \ldots
$$

It has two linearly independent systems of solutions:

$$
\begin{gathered}
P(\lambda):=\left(P_{n}(\lambda)\right)_{n=0}^{\infty} \quad \text { and } \quad Q(\lambda):=\left(Q_{n}(\lambda)\right)_{n=0}^{\infty} ; \\
P_{0}(\lambda)=1, P_{1}(\lambda)=\frac{\lambda-b_{0}}{a_{0}} ; \quad Q_{0}(\lambda)=0, Q_{1}(\lambda)=\frac{1}{a_{0}} .
\end{gathered}
$$


Thus, $P_{n}(\lambda)$ and $Q_{n}(\lambda)$ are polynomials in $\lambda$, and any solution of (9) is a linear combination of them. We write $P_{n}(\lambda)=p_{0, n}+p_{1, n} \lambda+\cdots+p_{n, n} \lambda^{n} ; p_{n, n}>0$. The polynomials $P_{n}(\lambda)$ are orthogonal with respect to $S($ or $d \rho(\lambda))$ :

$$
\sum_{i=0}^{n} \sum_{j=0}^{m} p_{i, n} p_{j, m} s_{i+j}=\int_{-\infty}^{\infty} P_{n}(\lambda) P_{m}(\lambda) d \rho(\lambda)=\delta_{m, n} .
$$

This can be proved in the following manner. From the definition of $J$ we get that $a_{n-1} e_{n-1}+b_{n} e_{n}+a_{n} e_{n+1}=J e_{n}$. Comparing these relations with (9), we find that $e_{n}=P_{n}(J) e_{0}$. Then, using (6), we have

$$
\begin{aligned}
& \delta_{m, n}=\left(e_{m}, e_{n}\right)=\left(P_{m}(J) e_{0}, P_{n}(J) e_{0}\right)=\left(P_{n}(J) P_{m}(J) e_{0}, e_{0}\right)= \\
& =\int_{-\infty}^{\infty} P_{n}(\lambda) P_{m}(\lambda) d \rho(\lambda) .
\end{aligned}
$$

Using the above relations, we get a solution of the inverse problem: given $\rho(\lambda)$ and the sequence of powers $1, \lambda, \lambda^{2}, \ldots$ we construct the polynomials $P_{n}(\lambda)$ with leading positive coefficients by applying the standard orthogonalization procedure. From this orthogonality and (9) follows

$$
a_{n}=\int_{-\infty}^{\infty} \lambda P_{n}(\lambda) P_{n+1}(\lambda) d \rho(\lambda), \quad b_{n}=\int_{-\infty}^{\infty} \lambda P_{n}^{2}(\lambda) d \rho(\lambda) .
$$

Also, the orthogonality relations lead to the representation of $P_{n}(\lambda)$ via the moments:

$$
P_{n}(\lambda)=\sqrt{\frac{1}{\Delta_{n-1}(J) \Delta_{n}(J)}}\left|\begin{array}{cccc}
s_{0} & s_{1} & \ldots & s_{n} \\
s_{1} & s_{2} & \ldots & s_{n+1} \\
\vdots & \vdots & \ldots & \vdots \\
s_{n-1} & s_{n} & \ldots & s_{2 n-1} \\
1 & \lambda & \ldots & \lambda^{n}
\end{array}\right|,
$$

Hence $P_{n}(\lambda)=\sqrt{\frac{\Delta_{n-1}(J)}{\Delta_{n}(J)}} \lambda^{n}+R_{n-1}(\lambda)$, where $R_{n-1}(\lambda)$ is some polynomial of degree $n-1$, and it follows from (9) that

$$
a_{n}=\frac{\sqrt{\Delta_{n+1}(J) \Delta_{n-1}(J)}}{\Delta_{n}(J)}, \quad b_{n}=\frac{D_{n}(J)}{\Delta_{n}(J)}-\frac{D_{n-1}(J)}{\Delta_{n-1}(J)},
$$

where

$$
D_{n}(J)=\operatorname{det}\left(\begin{array}{cccc}
s_{0} & \ldots & s_{n-1} & s_{n+1} \\
\vdots & & \vdots & \vdots \\
s_{n} & \ldots & s_{2 n-1} & s_{2 n+1}
\end{array}\right), \quad \Delta_{-1}=1, D_{-1}=0, D_{0}(J)=s_{1} .
$$

Thus, the equations (13) give the reconstruction procedure of the elements of $J$ in terms of the moment sequence $S$, and this procedure amounts to the solution (11) of the inverse spectral problem for the operator $J$. Given an arbitrary function $\rho(\lambda),-\infty<\lambda<\infty$, such that all its moments $s_{k}$ defined by (5) are finite and $s_{0}=1$; a solvability criterion for the inverse spectral problem for a certain operator $J$ can be formulated in two equivalent ways:

1. $\rho(\lambda)$ is a non-decreasing function having an infinite number of points of increase;

2. its moment sequence $S=\left\{s_{k}\right\}_{k=0}^{\infty}$ is positive, i. e. the condition (8) is fulfilled (the Hamburger's theorem).

Thus, the inverse spectral problem for $J$ can be formalized in terms of the associated moment sequence $S$, and its moments can be considered as the inverse spectral problem data.

Using the second of the equations (13) and applying induction on $n$ we obtain the following criterion of "sparsity" of the matrix $J$ in terms of $S$ :

Proposition 1. If $S$ is a moment sequence associated with $J$, then $\left\{b_{n}=0\right\}_{n=0}^{\infty} \Longleftrightarrow\left\{s_{2 k+1}=0\right\}_{k=0}^{\infty}$ 
As mentioned in the previous section, a reconstruction of the matrix $J$ with zero main diagonal may be considered as a solution of incomplete inverse problem for it, and the above result gives an additional condition on the elements of $S$ such that the required property of $J$ is fulfilled.

\subsection{Positive operator case}

Now consider the operators $J$ which satisfy the condition (2). First we establish that this condition is equivalent to (strict) positivity of the principal submatrices of the matrix $J$.

Lemma 1. The operator $J$ is positive, iff for all $N \geq 0$,

$$
\operatorname{det}\left(J_{N}\right)>0,
$$

where

$$
J_{N}=\left(\begin{array}{cccc}
b_{0} & a_{0} & 0 & 0 \\
a_{0} & b_{1} & a_{1} & 0 \\
0 & \ddots & \ddots & \ddots \\
0 & \ldots & a_{N-1} & b_{N}
\end{array}\right), \quad J_{0}=b_{0} .
$$

Proof. First assume that (14) is fulfilled. Take an arbitrary $y=\left(y_{n}\right)_{n=0}^{\infty} \in D(J)$ and the finitely nonzero vectors $y_{N}=\left(y_{0}, \ldots, y_{N}, 0,0, \ldots\right)$. Due to Sylvester's criterion $\left(J_{N} y_{N}, y_{N}\right)>0$, which in turn implies that $\left(J y_{N}, y_{N}\right)>$ 0 . Passing to the limit as $N \rightarrow \infty$ we get (2).

Conversely, assume that the operator $J$ is positive. We check directly that in this case $b_{0}>0$. Since the matrix $J$ has a three-diagonal structure, we get the following recurrence relation for determinants of $J_{N}$ :

$$
\operatorname{det}\left(J_{N+1}\right)=b_{N+1} \operatorname{det}\left(J_{N}\right)-a_{N}^{2} \operatorname{det}\left(J_{N-1}\right) .
$$

Assuming that $J_{0}, \ldots, J_{N-1}>0 ; J_{N}=0$, we find from (15) that $J_{N+1}<0$. Hence, there exists a vector $z_{N+1}=$ $\left(z_{0}, \ldots, z_{N+1}, 0,0, \ldots\right)$ such that $\left(J z_{N+1}, z_{N+1}\right)<0$, which contradicts (2) and thus (14) is established.

As follows from the proof, a necessary but not sufficient condition for the operator $J$ to be positive is that all $b_{N}>0$.

We now turn to the polynomials $P(\lambda)$ defined by (10) and orthogonal with respect to $S$. As follows from their definition, they satisfy the matrix equation

$$
J P(\lambda)=\lambda P(\lambda) .
$$

In particular, from this equation follows that the defect subspaces of the operator $J$ are at most onedimensional. Also, it follows from (16) that the vectors $\tilde{P}_{N}(\lambda):=\left(P_{0}(\lambda), \ldots, P_{N}(\lambda)\right)$ satisfy the equations

$$
\left(J_{N}-\lambda E_{N}\right) \tilde{P}_{N}=\tilde{R}_{N},
$$

where $\tilde{R}_{N}=(\overbrace{0, \ldots, 0,}^{N \text { times }}-a_{N} P_{N+1}(\lambda))$. Applying the Cramer's rule for calculating $P_{N}(\lambda)$, we find that

$$
P_{N+1}(\lambda)=-\frac{\operatorname{det}\left(J_{N}-\lambda E_{N}\right) P_{N}(\lambda)}{a_{N} \operatorname{det}\left(J_{N-1}-\lambda E_{N-1}\right)},
$$

and, consequently,

$$
P_{N+1}(\lambda)=(-1)^{N} \frac{\operatorname{det}\left(J_{N}-\lambda E_{N}\right) P_{1}(\lambda)}{a_{1} \ldots a_{N} \operatorname{det}\left(J_{0}-\lambda\right)} .
$$

Setting $\lambda=0$, we get

$$
P_{N+1}(0)=(-1)^{N+1} \frac{\operatorname{det}\left(J_{N}\right)}{a_{0} \ldots a_{N}}
$$


On the other hand, it follows from (12) that

$$
P_{N+1}(0)=(-1)^{N+1} \frac{E_{N}}{\sqrt{\Delta_{N} \Delta_{N+1}}} \text {, where } E_{N}=\operatorname{det}\left(s_{1+i+j}\right)_{i, j=0}^{N} .
$$

Hence,

$$
\operatorname{det}\left(J_{N}\right)=(-1)^{N+1} a_{0} \ldots a_{N} P_{N+1}(0)=a_{0} \ldots a_{N} \frac{E_{N}}{\sqrt{\Delta_{N} \Delta_{N+1}}}
$$

As follows from (13),

$$
a_{0} \ldots a_{N}=\sqrt{\frac{\Delta_{N+1}}{\Delta_{N}}}
$$

and we finally obtain

$$
\operatorname{det}\left(J_{N}\right)=\frac{E_{N}}{\Delta_{N}}
$$

Also note that the following formula for $\Delta_{N}$ can be derived from (13) and (17):

$$
\Delta_{N}=a_{0}^{2 N} a_{1}^{2(N-1)} \ldots a_{N-1}^{2} .
$$

Now let $S^{+}=\left\{s_{k}^{+}\right\}_{k=0}^{\infty}$ be the sequence such that $s_{2 k}^{+}=s_{k}, s_{2 k+1}^{+}=0$. We now check that the determinants $\Delta_{k}^{+}=: \operatorname{det}\left(H_{k}^{+}\right)=\operatorname{det}\left(\left(s_{i+j}^{+}\right)_{i, j=0}^{k}\right)$ admit a factorization in terms of $\Delta_{k}$ and $E_{k}$.

Lemma 2. The following representation holds for $\Delta_{k}^{+}$:

$$
\Delta_{k}^{+}=\Delta_{m} E_{m^{\prime}}, \quad k \in \mathbb{N}, \quad\left(\Delta_{0}^{+}=\Delta_{0}\right) ;
$$

where $m=\left[\frac{k}{2}\right], m^{\prime}=\left[\frac{k-1}{2}\right]$.

Proof. According to their definition, $H_{k}^{+}$are the following sparse Halkel matrices:

$$
H_{k}^{+}=\left(\begin{array}{ccccccc}
s_{0} & 0 & s_{1} & 0 & \ldots & \ldots & \\
0 & s_{1} & 0 & s_{2} & \ldots & \ldots & \\
s_{1} & 0 & s_{2} & 0 & s_{3} & \ldots & \ldots \\
\vdots & \ddots & \ddots & \ddots & \ddots & \ddots & \vdots \\
\ldots & \ldots & s_{k-3} & 0 & s_{k-2} & 0 & s_{k-1} \\
& \ldots & \ldots & s_{k-2} & 0 & s_{k-1} & 0 \\
& \ldots & \ldots & 0 & s_{k-1} & 0 & s_{k}
\end{array}\right),
$$

which have $m+1$ even rows (i. e. rows 0,2 , etc.) and $m^{\prime}+1$ odd ones. Successively applying transpositions first to their rows, and then to their columns (the total numbers of such transpositions are equal to each other), we rearrange the matrices to be made up of four blocks:

$$
\left(\begin{array}{cccc|cccc}
s_{0} & s_{1} & \ldots & s_{m} & & & & \\
s_{1} & s_{2} & \ldots & s_{m+1} & & 0_{m+1 \times m^{\prime}+1} & \\
\vdots & \vdots & \vdots & \vdots & & & & \\
s_{m} & s_{m+1} & \ldots & s_{2 m} & & & & \\
\hline & & & & s_{1} & s_{2} & \ldots & s_{m^{\prime}+1} \\
& & & & s_{2} & s_{3} & \ldots & s_{m^{\prime}+2} \\
& 0_{m^{\prime}+1 \times m+1} & & \vdots & \vdots & \vdots & \vdots \\
& & & & s_{m^{\prime}+1} & s_{m^{\prime}+2} & \ldots & s_{2 m^{\prime}+1}
\end{array}\right),
$$

and the desired representation follows immediately.

Now we are able to formulate the criterion for solvability of the inverse spectral problem for the positive operator $J$ in terms of the associated moment sequence. 
Theorem 1. A sequence $S=\left\{s_{k}\right\}_{k=0}^{\infty}, s_{k} \in \mathbb{R}$ is the associated moment sequence of a positive Jacobi operator $J$, iff it satisfies the following conditions:

1. $s_{0}=1$;

2. $s_{1}>0$;

3. for all $k \in \mathbb{Z}_{+}$the determinants

$$
\Delta_{k}^{+}>0 \text {. }
$$

Proof. First note that if the first two of the above conditions are fulfilled, the third one is equivalent to:

$$
\Delta_{k}>0, E_{k}>0 .
$$

This can be checked by using (19) and applying induction on $k$. Thus $S$ is a positive sequence, and this fact guarantees the existence of the corresponding operator $J$, such that $s_{k}=\left(J^{k}\right)_{0,0}$ and it remains to establish its positivity. As follows from Lemma 1, it suffices to show that the conditions (22) and (14) are equivalent. If (22) is fulfilled, then we obtain from (18) that the determinants $\operatorname{det}\left(J_{N}\right)$ are as well positive for all $N$. Conversely, if $\operatorname{det}\left(J_{N}\right)>0$ (in particular, $J_{0}=b_{0}=s_{1}>0$ ) then using (8) and (18) we find that $E_{k}>0$ thus establishing (22).

Note that the inverse spectral problem for positive operators $J$ (since their positiveness is a priori known) can be considered as an incomplete one, and the condition (22) which provides such property is more complicated than (8) for the general case of Jacobi operators.

Thus we obtained the criterion of positivity of $J$ in terms of the associated moment sequence. This criterion implies (see e. g. [20], Theorem 1) the existence of a function $\rho_{S t}(\lambda)$ which solves the Stieltjes moment problem for $S$ :

$$
s_{k}=\int_{0}^{\infty} \lambda^{k} d \rho_{S t}(\lambda) .
$$

If we take a spectral function $F_{t}$ of the positive operator $J$, one can check using the positivity of $J^{\star}$ and (3) that $F_{t}=O$ for $t \leq 0$ (wherein $F_{0_{+}}$is not necessarily a zero operator). Then, as in the general case, it can be established that each solution of the Stieltjes moment problem for the sequence $S$ corresponding to a positive operator $J$ can be described via spectral functions of this operator. Thus, in the same manner as the Hamburger moment problem is included into the theory of Jacobi operators, the Stieltjes moment problem can be included into the theory of their positive counterparts.

\section{Miura transformations between Kac - van Moerbeke and Toda lattices.}

\subsection{The lattices and their integration method}

The semi-infinite Kac-van Moerbeke (KvM) lattice is typically defined as the system

$$
\begin{gathered}
\dot{a}_{i}=a_{i}\left(a_{i+1}^{2}-a_{i-1}^{2}\right), \quad i \in \mathbb{Z}_{+}, \quad a_{i}=a_{i}(t), \quad t \in[0, T), \quad 0<T \leq \infty ; \\
a_{i}(t)>0, \quad a_{-1}=0, \quad \cdot=\frac{d}{d t} ;
\end{gathered}
$$

which appears as a result of transformation

$$
a_{i}(t)=\frac{1}{2} e^{-\frac{1}{2} R_{i}(t)}
$$


applied to the original system $[13,14,22]$

$$
\frac{d R_{i}(t)}{d t}=\frac{1}{2}\left(e^{-R_{i-1}(t)}-e^{-R_{i+1}(t)}\right) .
$$

The semi-infinite Toda lattice is a model for a one-dimensional chain of particles described by the system of equations

$$
\ddot{q}_{i}=e^{q_{i-1}-q_{i}}-e^{q_{i}-q_{i+1}}, \quad i \in \mathbb{Z}_{+}, \quad q_{i}=q_{i}(t), \quad t \in[0, T), \quad q_{-1}=0 ;
$$

where $q_{i}$ is the displacement of the $i$-th particle from its equilibrium position. In Flaschka's variables

$$
A_{i}=\frac{1}{2} e^{\frac{1}{2}\left(q_{i}-q_{i+1}\right)}, \quad B_{i}=-\frac{1}{2} \dot{q}_{i}
$$

it is written as

$$
\left\{\begin{array}{l}
\dot{A}_{i}=A_{i}\left(B_{i+1}-B_{i}\right) \\
\dot{B}_{i}=2\left(A_{i}^{2}-A_{i-1}^{2}\right) .
\end{array}\right.
$$

Both (23) and (24) admit the Lax representation $\dot{L}=[L, A]=L(t) A(t)-A(t) L(t)$ with

$$
L:=L_{k m}=\left(\begin{array}{ccccc}
0 & a_{0} & 0 & 0 & 0 \\
a_{0} & 0 & a_{1} & 0 & 0 \\
0 & a_{1} & 0 & a_{2} & 0 \\
0 & 0 & \ddots & \ddots & \ddots
\end{array}\right), A_{k m}:=\left(\begin{array}{ccccc}
0 & 0 & -a_{0} a_{1} & 0 & 0 \\
0 & 0 & 0 & -a_{1} a_{2} & 0 \\
a_{0} a_{1} & 0 & 0 & 0 & -a_{2} a_{3} \\
0 & a_{1} a_{2} & \ddots & \ddots & \ddots
\end{array}\right) ;
$$

for the system (23) and

$$
L:=L_{t o}=\left(\begin{array}{ccccc}
B_{0} & A_{0} & 0 & 0 & 0 \\
A_{0} & B_{1} & A_{1} & 0 & 0 \\
0 & A_{1} & B_{2} & A_{2} & 0 \\
0 & 0 & \ddots & \ddots & \ddots
\end{array}\right), A:=A_{t o}=\left(\begin{array}{ccccc}
0 & -A_{0} & 0 & 0 & 0 \\
A_{0} & 0 & -A_{1} & 0 & 0 \\
0 & A_{1} & 0 & -A_{2} & 0 \\
0 & 0 & \ddots & \ddots & \ddots
\end{array}\right) ;
$$

for (24) respectively. As we see, both $L_{k m}$ and $L_{t o}$ are Jacobi matrices, so the results of the previous section are applicable to the corresponding operators. In particular, both operators can be reconstructed from their inverse spectral data. The above observations indicate the following way for solving the initial boundary value problem for both lattices: first, calculate the inverse spectral data of the operators $L_{k m}(0)$ and $L_{t o}(0)$, then calculate the evolution in time of these data according to the Lax equation, and, finally, reconstruct the operators $L_{k m}(t)$ and $L_{t o}(t)$ from the inverse spectral data using (11) or (13). Such procedure was applied for solving this problem in the class of coefficients, uniformly bounded at each time, by Berezanski in [5]. In particular, it was established that if we denote as $m_{k m}(z, t), \rho_{k m}(\lambda, t)$, and

$$
S^{\prime}=\left\{s_{k}^{\prime}(t)\right\}_{k=0}^{\infty}, s_{k}^{\prime}(t)=\left(\left(L_{k m}(t)\right)^{k}\right)_{0,0},
$$

the spectral data of Jacobi operators $L_{k m}(t)$, and, respectively, as $m_{t o}(z, t), \rho_{t o}(\lambda, t)$, and

$$
\tilde{S}=\left\{\tilde{s}_{k}(t)\right\}_{k=0}^{\infty}, \tilde{s}_{k}(t)=\left(\left(L_{t o}(t)\right)^{k}\right)_{0,0}
$$

the spectral data of $L_{t o}(t)$, then their time evolution is described by the following equations:

$$
\begin{aligned}
\dot{m}_{k m}(z, t) & =2\left(m_{k m}(z, t)\left(z^{2}-a_{0}^{2}(t)\right)+z\right)=2\left(m_{k m}(z, t)\left(\left(z^{2}-s_{2}^{\prime}(t)\right)+z\right),\right. \\
d \dot{\rho_{k m}}(\lambda, t) & =2\left(\lambda^{2}-a_{0}^{2}(t)\right) d \rho_{k m}(\lambda, t)=2\left(\lambda^{2}-s_{2}^{\prime}(t)\right) d \rho_{k m}(\lambda, t), \\
\frac{d s_{2 k}^{\prime}(t)}{d t} & =2\left(s_{2(k+1)}^{\prime}-s_{2}^{\prime} s_{2 k}^{\prime}\right), \quad k \in \mathbb{Z}_{+} ;
\end{aligned}
$$

and

$$
\begin{aligned}
\dot{m}_{t o}(z, t) & =2\left(m_{t o}(z, t)\left(\left(z-B_{0}(t)\right)+1\right)=2\left(m_{t o}(z, t)\left(\left(z-\tilde{s}_{1}(t)\right)+1\right)\right.\right. \\
d \dot{\rho}_{t o}(\lambda, t) & =2\left(\lambda-B_{0}(t)\right) d \rho_{t o}(\lambda, t)=2\left(\lambda-\tilde{s}_{1}(t)\right) d \rho_{t o}(\lambda, t) \\
\frac{d \tilde{s}_{k}(t)}{d t} & =2\left(\tilde{s}_{k+1}-\tilde{s}_{1} \tilde{s}_{k}\right), \quad k \in \mathbb{Z}_{+}
\end{aligned}
$$


Note that since the matrices $L_{k m}(t)$ have zero main diagonal elements, it follows from the Proposition 1 that the moments $s_{2 k+1}^{\prime}(t)=0, k \in \mathbb{Z}_{+}$. In [4], the equations for the Weyl functions were derived from the evolution of polynomials $P(\lambda, t), Q(\lambda, t)(10)$ corresponding to the matrices $L(t)$. After that, the equations for $d \rho(\lambda, t)$ were obtained using the uniqueness of the Stieltjes transform (4) between the latter and $m(z, t)$. The equations for the moments were then easily obtained. The second equations in (29) and (30) lead to the following formulas for the corresponding measures.

$$
d \rho_{k m}(\lambda, t)=\frac{e^{2 \lambda^{2} t} d \rho_{k m}(\lambda, 0)}{\int_{-\infty}^{\infty} e^{2 \lambda^{2} t} d \rho_{k m}(\lambda, 0)} ; \quad d \rho_{t o}(\lambda, t)=\frac{e^{2 \lambda t} d \rho_{t o}(\lambda, 0)}{\int_{-\infty}^{\infty} e^{2 \lambda t} d \rho_{t o}(\lambda, 0)} .
$$

Both of them were obtained with the use of normalization condition for the measures, for example, as follows from (29)

$$
d \rho_{k m}(\lambda, t)=e^{2 \lambda^{2} t} d \rho_{k m}(\lambda, 0) e^{\left.-\int_{0}^{t} 2 a_{0}^{2}(\tau)\right) d \tau},
$$

then we find

$$
1=\int_{-\infty}^{\infty} d \rho_{k m}(\lambda, t)=e^{\left.-\int_{0}^{t} 2 a_{0}^{2}(\tau)\right) d \tau} \int_{-\infty}^{\infty} e^{2 \lambda^{2} t} d \rho_{k m}(\lambda, 0) .
$$

The latter relation leads to the representation (31) for $d \rho_{k m}(\lambda, t)$. The formula for $d \rho_{t o}(\lambda, t)$ can be found similarly.

If the elements of Jacobi matrix are uniformly bounded, then the corresponding operator $J$ is bounded and therefore self-adjoint. This in particular means that it has a unique spectral function which coincides with its spectral measure and that the corresponding function $\rho(\lambda)$ is compactly supported on $\mathbb{R}$. It follows from (31) that if at initial time the operators $L_{k m}(0)$, and $L_{t o}(0)$ are bounded, then at any time $t$ the measures $d \rho_{k m}(\lambda, t)$ and $d \rho_{t o}(\lambda, t)$ have a compact support uniformly with respect to $t$. Since $L_{k m}(0)$ is a positive operator, the measures $d \rho_{k m}(\lambda, t)$ are compactly supported on $\mathbb{R}_{+}$. Then, from the orthonormality of $P(\lambda, t)$ and (11) follows that the elements of the matrices $L_{k m}(t)$ and $L_{t o}(t)$ are uniformly bounded, which imply the boundedness of the corresponding Jacobi operators. Thus, the initial boundary value problem for both systems (23) and (24) is solvable in a class of sequences bounded at every time value. In the non-bounded case (even if the initial Jacobi operator is not self-adjoint) the existence of solutions of (23) and (24) follows from convergence of the integrals $\int_{-\infty}^{\infty} e^{2 \lambda^{2} t} d \rho_{k m}(\lambda, 0)$ and $\int_{-\infty}^{\infty} e^{2 \lambda t} d \rho_{t o}(\lambda, 0)$ (which are the denominators in the measures $d \rho_{k m}(\lambda, t)$ and $d \rho_{t o}(\lambda, t)$ given by (31)) on [0,T). If the measure $d \rho_{t o}(\lambda, 0)$ is supported on the positive half line, which means that the corresponding operator $L_{t o}(0)$ is positive, then this is as well true for $d \rho_{t o}(\lambda, t)$ and $L_{t o}(t)$.

\subsection{Direct and inverse discrete Miura transformations.}

The discrete Miura transformation $M d$ between the systems (23) and (24) is defined as follows:

$$
M d:\left(a_{i}\right)_{i=0}^{\infty} \rightarrow\left(A_{i}, B_{i}\right)_{i=0}^{\infty} ; \quad A_{i}=a_{2 i} a_{2 i+1}, \quad B_{i}=a_{2 i}^{2}+a_{2 i-1}^{2} .
$$

It provides a relation between the KvM and the Toda lattices in the way that the transformation (1) relates the modified Korteweg-de Vries equation to the original KdV. In particular, using (32) one can derive the $\mathrm{N}$ soliton solutions of the both systems starting from the trivial ones [11, 23].

Due to the Lax representations (25) and (26), we can associate the solutions of (23) and (24) to the moment sequences $S^{\prime}$ (27) of $L_{k m}(t)$ and, respectively, $\tilde{S}(28)$ of $L_{t o}(t)$. In [19] Theorem 4, a simple description of the transformation $M d$ in terms of $S^{\prime}$ and $\tilde{S}$ was established:

$$
M d: S^{\prime} \rightarrow \tilde{S}, \quad \tilde{s}_{k}(t)=s_{2 k}^{\prime}(t), \quad k \in \mathbb{Z}_{+} .
$$

(in fact, in [19] both lattices were studied using the non-symmetric matrices $L$ in their Lax representation, so the result of the Theorem 4 has to be slightly modified to fit the above case of (25)-(26)).

Thus, due to (32), to each KvM lattice there corresponds a Toda lattice with positive coefficients $B_{i}$. Obviously, $M d$ does not provide a bijection between the systems (23) and (24) and, therefore, between $S^{\prime}$ and $\tilde{S}$. 
The inverse Miura transformation IMd is defined recurrently:

$$
\text { IMd }:\left(A_{i}, B_{i}\right)_{i=0}^{\infty} ; \rightarrow\left(a_{i}\right)_{i=0}^{\infty} ; \quad a_{2 i}=\sqrt{B_{i}-a_{2 i-1}^{2}}, \quad a_{2 i+1}=\frac{A_{i}}{a_{2 i}}, \quad i \in \mathbb{Z}_{+} .
$$

For $i>0$, the formulas for $a_{2 i}^{2}$ can be written as finite continued fractions:

$$
a_{2 i}^{2}=B_{i}-\frac{A_{i-1}^{2}}{B_{i-1}-\frac{A_{i-2}^{2}}{B_{i-2}-\frac{A_{i-3}^{2}}{\ddots-\frac{A_{0}^{2}}{B_{0}}}} .}
$$

As follows from (34),

$$
a_{2 i}^{2}=B_{i}-\frac{A_{i-1}^{2}}{a_{2 i-2}^{2}} .
$$

Comparing the latter formula with (15) we find that $a_{2 i}^{2}$ and $\frac{\operatorname{det}\left(J_{N}\right)}{\operatorname{det}\left(J_{N-1}\right)}$ satisfy the same recurrent relations, from which we derive

$$
a_{2 i}^{2}=\frac{\operatorname{det}\left(J_{i}\right)}{\operatorname{det}\left(J_{i-1}\right)}
$$

here

$$
J_{i}=\left(\begin{array}{cccc}
B_{0} & A_{0} & 0 & 0 \\
A_{0} & B_{1} & A_{1} & 0 \\
0 & \ddots & \ddots & \ddots \\
0 & \ldots & A_{i-1} & B_{i}
\end{array}\right), \quad J_{0}=B_{0}, \quad J_{-1}=1 .
$$

Thus, the transformation IMd maps an arbitrary solution $\left(A_{i}(t), B_{i}(t)\right)_{i=0}^{\infty}$ of (24) into a solution $\left(a_{i}(t)\right)_{i=0}^{\infty}, a_{i}(t)>0$ of $(23)$ only if the determinants $J_{N}$ composed of $\left(A_{i}(t), B_{i}(t)\right)$ are positive. According to the Lemma 1, the latter imply that the corresponding operator $L_{t o}(t)$ is positive. As follows from the Theorem 1, this property of $L_{t o}(t)$ can be formulated via the associated moment sequence $\tilde{S}$.

We say that (24) is a positively defined Toda lattice if it has a solution $\left(A_{i}, B_{i}\right)_{i=0}^{\infty}$ such that the matrix $L_{t o}$ (26) generates the positive Jacobi operator. In view of the above considerations, we obtain:

Theorem 2. The direct (32) and the inverse (34)-(35) discrete Miura mappings provide a bijection between the set of KvM lattices (23) and the set of positively defined Toda lattices (24). In terms of the moment sequences (27) and (28), related to KvM and Toda lattices respectively, the direct transform is given by (33), while the inverse one is given by:

$$
I M d: \tilde{S} \rightarrow S^{\prime}, \quad s_{2 k}^{\prime}(t)=\tilde{s}_{k}(t) ; \quad s_{2 k+1}=0, \quad k \in \mathbb{Z}_{+} .
$$

\section{Discussion and conclusions}

As noted above, the study of nonlinear equations and their integration methods made a significant impact on operator theory. It has to be pointed out that this influence is not restricted to the inverse problems for differential and difference operators.

Here we show how the Miura transformations can be applied to the study of self-adjointness of Jacobi operators. In doing so we use the relations between the theory of the latter and the power moment problems. First, we need to establish a connection between the determinate (i. e. having a unique solution) Hamburger and Stieltjes moment problems. Namely, combining the Proposition 1.6 of [20] with the Theorem 2.13 of the same paper, one gets the following result.

Proposition 2. Let $\left\{S t_{k}\right\}_{k=0}^{\infty}$ be a set of Stieltjes moments. Let

$$
\Gamma_{2 k}=S t_{k}, \quad \Gamma_{2 k+1}=0, \quad k \in \mathbb{Z}_{+} .
$$


Then $\left\{\Gamma_{k}\right\}_{k=0}^{\infty}$ is a determinate Hamburger problem if and only if $\left\{S t_{k}\right\}_{k=0}^{\infty}$ is a determinate Stieltjes problem.

As known, (see e. g. [1]) the determinacy of a Hamburger or a Stieltjes moment problem implies that the corresponding Jacobi operator is self-adjoint (in the latter case it is also positive). Now, take two operators, $J_{1}$ and $J_{2}$, generated by the matrices

$$
J_{1}=\left(\begin{array}{ccccc}
0 & a_{0} & 0 & 0 & 0 \\
a_{0} & 0 & a_{1} & 0 & 0 \\
0 & a_{1} & 0 & a_{2} & 0 \\
0 & 0 & \ddots & \ddots & \ddots
\end{array}\right), J_{2}=\left(\begin{array}{ccccc}
B_{0} & A_{0} & 0 & 0 & 0 \\
A_{0} & B_{1} & A_{1} & 0 & 0 \\
0 & A_{1} & B_{2} & A_{2} & 0 \\
0 & 0 & \ddots & \ddots & \ddots
\end{array}\right) ;
$$

and assume that they are related to each other by Miura transformations (32)and (34); we denote that as $J_{2}=M d\left(J_{1}\right)$, and $J_{1}=I M d\left(J_{2}\right)$. The application of these operators to the integration of nonlinear lattices is not necessarily assumed. Then, if we denote as $S^{\prime}$ and $\tilde{S}$ the associated moment sequences of $J_{1}$ and $J_{2}$ respectively, then, according to the Theorem 2, these sequences are related to each other via (33) and (36). Comparing the latter with (37) and taking the above arguments into account, we find that if the former operator is self-adjoint then so is the latter one, and vice versa. More generally, the following result holds:

Theorem 3. Assume that $J_{2}=M d\left(J_{1}\right)$ and $J_{1}=I M d\left(J_{2}\right)$, where $J_{1}$ and $J_{2}$ are Jacobi operators corresponding to the matrices (38). Then for arbitrary bounded symmetric operators $A$ and $B$ defined on all $l^{2}$ and for arbitrary $a, b \in \mathbb{R}, a, b \neq 0$, the operator $\tilde{J}_{1}=a J_{1}+A$ is self-adjoint, iff so is the operator $\tilde{J}_{2}=b J_{2}+B$.

Here we use the invariance of deficiency indices of symmetric operators under bounded symmetric perturbations. As an example, consider the operator $J_{1}$ such that $a_{i}=i+1, i=0,1, \ldots$ We have, $\sum_{i} \frac{1}{a_{i}}=\infty$, therefore by the Carleman condition $J_{1}$ is self-adjoint. As follows from the above theorem, the operator $J_{2}=M d\left(J_{1}\right)$, generated by matrix $J_{2}$ (38) with the entries $B_{i}=(2 i)^{2}+(2 i+1)^{2}, A_{i}=(i+1)(i+2)$ is self-adjoint too, in spite of the fact that several known self-adjointness conditions (e. g. that of Carleman or Dennis-Wall, see $[1,18]$ for details) are not satisfied here.

As to the theory of integrable systems, as noted in [8], where the finite KvM (or Volterra) and Toda lattices were studied, the former one is dynamically and algebraically equivalent to the latter and the discrete Miura transformations provide this equivalence. In particular this means that they allow one to transform the Poisson brackets, master symmetries, first integrals of a system of one type to the similar objects of the system of the other type. For example, as shown in [10], the mapping (32) transforms the linear Poisson bracket for the KvM lattice to the linear Poisson bracket of its Toda counterpart, and it should be pointed out that the first of the brackets is non local, whereas the second one is local.

In the finite lattice case, due to the special structure of the moment sequence corresponding to a finite Jacobi operator and the equations (29) and (30) for the moments, one can find non-trivial first integrals for both KvM and Toda lattices, see [19] for the details. The relations (33) and (36) allow one to transform these first integrals into each other. The study of Poisson structures of both lattices using the Theorem 2, as well as the study of another nonlinear integrable dynamical systems via the corresponding inverse spectral data are subjects of our current research.

Acknowledgement: This work is done at FSU SRISA RAS according to the project No 0580-2021-0007.

Conflict of interest: Authors state no conflict of interest.

\section{References}

[1] N.I. Akhiezer, The Classical Moment Problem, Oliver Boyd, Edinburgh, (1965).

[2] N.I. Akhiezer, I.M. Glazman, Theory of Linear Operators in Hilbert Space, Dover Publications, New. York (1993). 
[3] A. I. Aptekarev, A. Branquinho, and F. Marcellan, Toda-type differential equations for the recurrence coeffcients of orthogonal polynomials and Freud transformation, J. Comput. Appl. Math. 78, (1997), 139-160.

[4] Ju. M. Berezanskij, Expansions in Egenfunctions of Selfadjoint Operators, AMS, Providence, R.I. (1968).

[5] Yu. M. Berezanski, The integration of semi-infinite Toda chain by means of inverse spectral problem. Repts. Math. Phys., vol. 24, No 1, (1986), 21-47.

[6] D. Barrios Rolaniá, A. Branquinho, A. Foulquié Moreno, Dynamics and interpretation of some integrable systems via multiple orthogonal polynomials. Journ. Math. An. Appl., vol. 361, issue 2, (2010), 358-370.

[7] C. Gardner, J. Greene, M. Kruskal, R. Miura Method for Solving the Korteweg de Vries Equation. Phys. Rev. Lett., 19, (1967), 1095-1097.

[8] P. Damianou, The Volterra model and its relation to the Toda lattice, Physics Lett. A, vol. 155, (1991), 126-132.

[9] J. Dombrovski, Cyclic operators, commutators, and absolutely continuous measures, Proc. Amer. Math. Soc., vol. 100, No 3, (1987), 457-463.

[10] L. Faybusovich, M. Gekhtman, On Schur flows, J. Phys. A: Math. Gen., 32, (1999), 4671-4680.

[11] F. Gesztesy, H. Holden, B. Simon, Z. Zhao, On the Toda and Kac-van Moerbeke systems, Trans. Amer. Math. Soc., 339 (2), (1993), 849-868.

[12] S. V. Manakov, Complete integrability and stochastization of discrete dynamical systems, Sov. Phys. JETP, vol. 40, issue 2, (1975), 269-274.

[13] Kac, P. van Moerbeke, On an explicitly soluble system of nonlinear differential equations related to a certain Toda lattice. Advances in Mathematics, vol. 16, (1975), 160-169.

[14] J. Moser, Three integrable Hamiltonian systems connected with isispectral deformations. Advances in Math.,vol. 16, (1975), 197-220.

[15] M.A. Naimark, Linear Differential Operators, Part II, Frederick Ungar Publishing Company, N. Y. (1968).

[16] S. Novikov, S.V. Manakov, L.P. Pitaevskii, V.E. Zakharov, Theory of Solitons: The Inverse Scattering Method (Monographs in Contemporary Mathematics), Berlin; New York: Springer-Verlag, (1984).

[17] A. Osipov, On some issues related to the moment problem for the band matrices with operator elements, Journ. Math. An. Appl., vol. 275, no. 2, (2002), 657-675.

[18] A. Osipov, On unbounded commuting Jacobi operators and some relater issues. Concrete Operators, vol. 6, No. 1, (2019), 82-91.

[19] Osipov A. S., Inverse Spectral Problems for Second-Order Difference Operators and Their Application to the Study of Volterra Type Systems, Rus. J. Nonlin. Dyn., vol. 16, no. 3, (2020), 397-419.

[20] B. Simon, The classical moment problem as a self-adjoint finite difference operator, Adv. Math. 137, (1998), 82-203.

[21] Yu.B. Suris, The Problem of Integrable Discretization: Hamiltonian Approach. Progress in Mathematics, vol. 219. Basel: Birkhäuser, (2003).

[22] M. Toda, Theory of nonlinear lattices, Berlin; New York: Springer-Verlag, (1981).

[23] G. Teschl, Jacobi Operators and Completely Integrable Nonlinear Lattices, Mathematical Surveys and Monographs, vol. 72, RI: AMS, (2000).

[24] V.A. Yurko, Inverse Spectral Problems for Linear Differential Operators and Their Applications (1st ed.), CRC Press, (2000).

[25] V. E. Zakharov, L. D. Faddeev, Korteweg-de Vries equation: A completely integrable Hamiltonian system, Funct. Anal. Its Appl., 5, (1971), 280-287. 